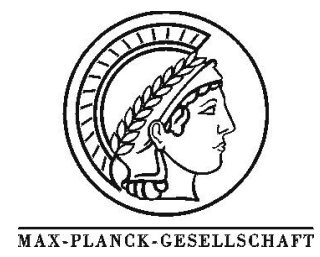

\title{
Photoinduced Decomposition of Nitrate in Drinking Water in the Presence of Titania and Humic Acids
}

\author{
Bettina Bems, Friederike C. Jentoft* and Robert Schlögl
}

Department of Inorganic Chemistry, Fritz-Haber-Institute of the MPG, Faradayweg 4-6, 14195 Berlin, Germany

"Corresponding author: e-mail jentoft@ fhi-berlin.mpg.de

Received 19 April 1998; accepted 18 October 1998

\begin{abstract}
The photoreduction of nitrate in aqueous medium was investigated at $292 \mathrm{~K}$ in a batch system open to the ambient. Titania was tested as a photocatalyst and humic acids were added as promoters. Conversions of $28 \%$ were reached after 80 hours when a $44 \mathrm{mg} / \mathrm{l}$ nitrate solution was irradiated with a high pressure Xe-lamp; the major product was nitrite. The addition of humic acids $(20 \mathrm{mg} / \mathrm{l})$ promoted reduction of nitrate to nitrite but the mechanism of promotion could not be unambiguously identified. Titania $(0.1 \mathrm{~g} / \mathrm{l})$ itself did not catalyze the photoreduction of nitrate but rather seemed to act as a catalyst for the reoxidation of nitrite to nitrate. The most successful system was a combination of $44 \mathrm{mg} / \mathrm{l}$ nitrate, $20 \mathrm{mg} / \mathrm{l}$ humic acids and $0.1 \mathrm{~g} / \mathrm{l}$ Kronos-1002 titania: the nitrate conversion reached $32 \%$ after 76 hours, with little nitrite formed. Photocatalytic nitrate degradation is accompanied by homogeneous reduction to the more toxic nitrite; requiring any effective catalyst system to also reduce nitrite concentration.
\end{abstract}

Keywords: titania, humic acids, nitrate, nitrite, photocatalysis, drinking water

\section{Introduction}

Ground water, one of the major sources of drinking water, is often contaminated with nitrate, particularly in intensively farmed agricultural regions. Nitrate and its metabolites are toxic for the human body, and can be particularly harmful to infants. The World Health Organization recommends a maximum nitrate concentration of $10 \mathrm{mg}$ nitrogen/l in drinking water. Various processes for the degradation of nitrate in drinking water have been developed [1] and current technologies include ion exchange, biodegradation, and reverse osmosis. These methods are either expensive, sensitive to reaction conditions or unsuitable for large scale implementation.
The restrictions on a catalytic process for nitrate removal from drinking water are severe, i.e. the product stream must conform to drinking water standards and energy consumption must be low for the process to be cost effective. The temperature and $\mathrm{pH}$ cannot be adjusted freely, and no toxic substances may leach from the catalyst. Photoinduced processes, initiated by sunlight, are thus a potential alternative to the aforementioned technologies. The photoreduction of nitrate in aqueous solution was reported as early as 1907 by Thiele [2]. Without catalyst, the toxic nitrite seems to be the major product, although the literature is not entirely consistent with regard to the product distribution and a possible nitrate/nitrite equilibrium [3-7]. 
Titania is a widely applied photocatalyst [8] which has been used for water treatment [9], predominantly for the oxidative destruction of non-biodegradable organic compounds [10-13]. Photoreduction in aqueous media in the presence of titania has been reported, e.g. the reduction of benzoquinones to hydrobenzoquinones [14]. Another example is the photocatalytic decomposition of nitrobenzene which yielded a small fraction of reduction products [15]. Simple inorganic ions were reduced with noble metal promoted titania, e.g. carbonate with photoplatinized titania as a catalyst [16]. Ranjit et al. [17] investigated the photoreduction of nitrate in an aqueous medium in the presence of ruthenium supported on titania, and reported that nitrate was successfully converted with ammonia as the major product. Kudo et al. [18,19] used a variety of systems for the photoreduction of nitrate, and reported that platinum supported on titania and platinum supported on strontium titanate were particularly successful catalysts. The use of the costly and potentially toxic noble metals could be avoided by (i) finding an alternative way to promote titania or (ii) providing reduction equivalents. The photochemical properties of humic acids allow for both these possibilities.

Humic acids are the base-soluble fraction of soils [20,21]. They are formed from dead plant fibers and are thus often present in natural waters. Humic acids are composed of $\mathrm{C}$, $\mathrm{H}, \mathrm{N}, \mathrm{O}$ and $\mathrm{S}$. Few humic acids have been identified and structurally characterized, but humic acids are classified as oxycarbonic acids. Frequently occurring functional groups are: hydroxyl, carboxyl, carbonyl, ether bridges, methoxy, amino, and heterocyclic nitrogen. Aromatic structure elements are typical, but aliphatic structure elements are also found. Humic acids are known to form different reactive species under irradiation with light in the UV/Vis range, among them singlet oxygen, superoxide, hydroxyl radicals and solvated electrons [22-25]. Some of these species, i.e. solvated electrons, are potential reducing agents. Additionally, electrons from excited humic acids can be transferred to the conduction band of titania [25]; these electrons could then be available for nitrate reduction. Humic acids may therefore be 'natural' promoters for photoreduction, and are employed here in concentrations which are typical for lakes and rivers (up to 25-30 mg/l [26]).

The scope of this paper was to assess the feasibility of a photocatalytic nitrate reduction process, employing titania and humic acids under conditions which could be suitable for a sun-light driven water purification unit. We have tested three different titania powders in an open reactor system, at ambient temperature and pressure, and without pH-modification. A high pressure Xe-lamp was used to approximate the emission spectrum of the sun. Quantitative nitrate analysis was performed according to an approved German industrial standard procedure.

\section{Experimental}

Chemicals: Purified water was obtained by double distillation of tap water. The tested titanias were P25 (Degussa AG, Hanau, Germany), Kronos-1002 (Kronos Titan-GmbH, Leverkusen, Germany), and DT-51 (RhonePoulenc, Mulhouse, France). The phase composition of P25 is $70 \%$ anatase and $30 \%$ rutile, and the surface area is $50 \mathrm{~m}^{2} / \mathrm{g}$ [27]. Kronos-1002 and DT-51 are $100 \%$ anatase and their surface areas were determined to be $9 \mathrm{~m}^{2} / \mathrm{g}$ and 90 $\mathrm{m}^{2} / \mathrm{g}$, respectively. Humic acids (Roth, Karlsruhe, Germany, Batch no. 485 18307) were employed in the form of the sodium salt for better solubility. The content of humic acids in this particular batch was $50.2 \%$.

Reactor System: The photocatalytic experiments were performed in an open batch system. An all-glass (Duran ${ }^{\circledR}$ ) reaction vessel of cylindrical shape with double walls was used. The ID was $80 \mathrm{~mm}$ and the inner height was $40 \mathrm{~mm}$. The temperature, which was monitored with a thermocouple in a glass well, was kept constant to within $\pm 1 \mathrm{~K}$ by flowing water between the reactor walls. The reactor was either wrapped in aluminum foil (dark experiments) or it was irradiated from the top. The horizontal beam from a $150 \mathrm{~W}$ Xe-high pressure lamp (Oriel, Darmstadt, Germany) was redirected into the reactor with a planar mirror. The reaction vessel was covered with a $3 \mathrm{~mm}$ thick quartz disk in order to minimize evaporation. The photon flux in the reactor was sufficient to convert the $\mathrm{Fe}(\mathrm{III})$ in $0.24 \mathrm{mmol}(40 \mathrm{ml} / 0.006 \mathrm{M})$ potassium ferrioxalate [28] in less than 60 seconds to $\mathrm{Fe}(\mathrm{II})$.

Reactions were performed at $292 \mathrm{~K}$ and atmospheric pressure, and the reaction medium was mixed with a magnetic stirrer. The reaction mixtures were prepared as follows (all components optional): An amount of titania, calculated to give a concentration of 0.1 or $0.05 \mathrm{~g} / \mathrm{l}$, was introduced into the reactor. A nitrate solution in water of the desired concentration was prepared by dilution of a stock solution $(110 \mathrm{mg} / \mathrm{l}$ nitrate). Humic acids were added to the nitrate solution (alternatively: water) in an amount as to obtain a final concentration of $20 \mathrm{mg} / \mathrm{l}$. The aqueous phase was then introduced into the reactor. A typical reaction mixture contained $44 \mathrm{mg} / \mathrm{l}$ nitrate, $20 \mathrm{mg} / \mathrm{l}$ humic acids and $0.1 \mathrm{~g} / \mathrm{l}$ titania. The mixture was stirred for about 5 min. until a homogeneous suspension was formed. At this point, an aliquot of $5 \mathrm{ml}$ was taken, and this sample was designated as $t=0$. The amount of evaporated water was determined at the end of the run and replaced before analysis. Conversion levels at different reaction times were determined from different runs (one data point per run).

Analysis of Reaction Mixture: Quantitative nitrate analysis was performed according to DIN 38 405-D9-2, a German industrial standard procedure. 2,6-Dimethylphenol and nitrate react in an acidic medium to give 4-nitro-2,6dimethylphenol, the concentration of which was determined photometrically. A commercially available spectrophotometric cuvette-test (Spektroquant ${ }^{\circledR}$ Nitrat Küvettentest, Merck, Darmstadt, Germany), based on the same DIN-procedure, was used. The sample and the 
reagent were placed in a cuvette, and after 10 minutes the absorption at $340 \mathrm{~nm}$ was measured (Perkin Elmer lambda 9). The concentrations were determined by calibration curves.

The humic acids did not dissolve entirely in water, and titania gave a suspension. Scattering and absorption due to humic acids and titania interfered with the cuvette test (Figure 1), complicating accurate nitrate determination in this matrix. Titania was removed by pressing the liquid through a membrane filter $(0.22 \mu \mathrm{m}$, Roth), as seen in Figure 1. Filtration was found to be faster and easier than centrifugation although the filters had to be washed before use because of leaching problems. The matrix effect of the humic acids was dealt with by establishing a second calibration line in the presence of humic acids. The confidence interval for the determination of the nitrate concentration was estimated to be $\pm 2-3 \mathrm{mg} / \mathrm{l}$, in the presence of humic acids it was $\pm 3-4 \mathrm{mg} / \mathrm{l}$.

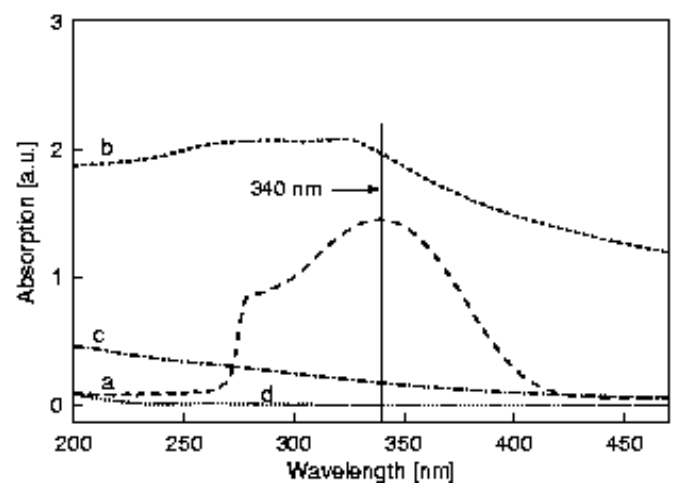

Figure 1: Interference of humic acids and titania with nitrate analysis. UV/Vis spectra of a) nitrate analysis (88 $\mathrm{mg}$ nitrate/l) with cuvette test, absorption is taken at 340 $\mathrm{nm}$; b) titania (P25) $0.1 \mathrm{~g} / \mathrm{l}$ in water; c) humic acid: $10 \mathrm{mg} / \mathrm{l}$ in water; d) titania (P25) $0.1 \mathrm{~g} / \mathrm{l}$ in water after filtration with $0.22 \mu \mathrm{m}$ membrane filter.

Reaction mixtures were also analyzed for products. If titania was present, the samples were filtered before analysis. The nitrite concentration was estimated with test strips (Merckoquant ${ }^{\circledR}$ Nitrit-Test, Merck) which indicate concentrations of $2,5,10,20,40$, or $80 \mathrm{mg} / \mathrm{l}$. The presence of ammonium was qualitatively analyzed for with Nessler's reagent (Merck, detection limit $5^{*} 10^{-8} \mathrm{~g} / \mathrm{ml}$ [29]). UV/Visspectra were recorded with a PE lambda 9 spectrometer.

\section{Results}

Nitrate, humic acids, and titania in water were investigated separately and in all possible combinations in order to distinguish the effects of the individual components from effects based on the interaction of the components. All experiments were carried out with irradiation and without irradiation (dark experiments) to identify light-induced effects. Whenever nitrate and/or humic acids were part of the initial reaction mixture, nitrite and ammonium analysis were performed after reaction. The ammonium test was never positive. UV/Vis spectra were used to check for the presence of nitrate in mixtures which initially did not contain nitrate.

The double-distilled water had a $\mathrm{pH}$ of 5.3. Nitrate reacted neutral, giving a $\mathrm{pH}$ of 5.7 at $44 \mathrm{mg} / \mathrm{l}$. Humic acids reacted slightly basic ( $\mathrm{pH}=7.4$ at $20 \mathrm{mg} / \mathrm{l})$, and titania slurries had $\mathrm{pH}$ values of 5.5 (P25) and 6.0 (Kronos) at $0.1 \mathrm{~g} / \mathrm{l}$. The typical reaction mixture was almost neutral $(\mathrm{pH}=7.2)$.

\section{Single component systems}

In the system containing only nitrate, nitrate was recovered to $100 \%$ in a 30 -hour dark experiment but decomposition of nitrate was observed under irradiation. Conversions reached $24 \%$ after 30 hours and $28 \%$ after 80 hours (initial nitrate concentration: $44 \mathrm{mg} / \mathrm{l}$ ) as shown in Figure 2. The nitrite concentration after 80 hours was $10 \mathrm{mg} / \mathrm{l}$.

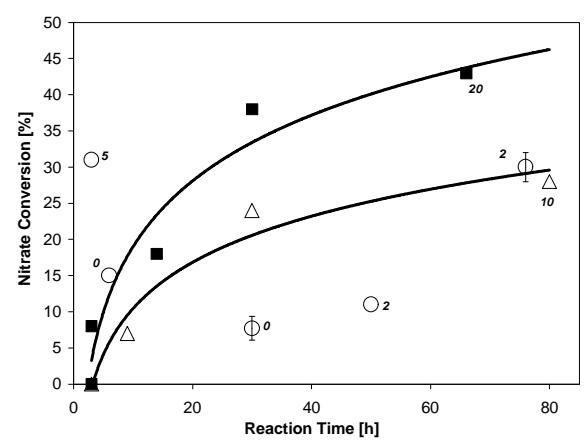

Figure 2: Nitrate conversion after irradiation vs. reaction time: Nitrate $44 \mathrm{mg} / \mathrm{l}$; humic acids $20 \mathrm{mg} / \mathrm{l}$; titania (Kronos-1002) $0.1 \mathrm{~g} / \mathrm{l}$. Symbols: $\Delta$ pure nitrate solution; in the presence of humic acids; $\mathrm{X}$ in the presence of humic acids and titania (Kronos-1002). Numbers indicate mg/l nitrite formed.

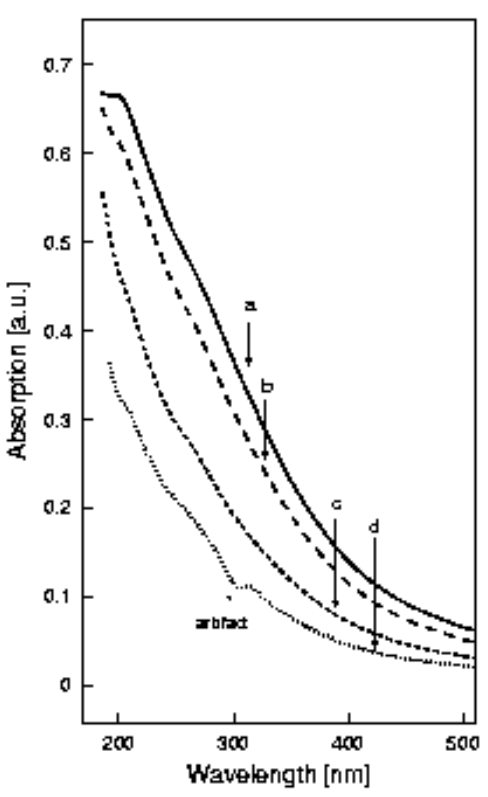

Figure 3: UV/Vis spectra of humic acids in aqueous phase after irradiation for a) 0 ; b) 3 ; c) 16 ; d) 30 hours. Humic acids $20 \mathrm{mg} / \mathrm{l}$. 
In the system containing only humic acids, UV/Vis spectra showed no changes in dark experiments but the spectra taken from irradiated mixtures were altered. A gradually decreasing absorption with irradiation time was observed, Figure 3.

In the system containing only titania, UV/Vis spectra were recorded from filtrates of titania suspensions which were reacted for 3 hours with and without irradiation. No significant changes were detected.

\section{Two component systems}

There were no detectable changes in the humic acid/nitrate system after the dark experiments. The nitrate concentration decreased rapidly when the nitrate/humic acid system was irradiated. Conversions, compared at the same reaction times, were higher than in the pure nitrate system, reaching $38 \%$ after 30 hours and $43 \%$ after 66 hours, Figure 2. Nitrite was detected in larger quantities $(20 \mathrm{mg} / \mathrm{l}$ at $38 \%$ conversion) than in the pure nitrate system (at slightly different nitrate conversion levels, i.e. $38 \%$ vs. $28 \%$ ).

For the nitrate/titania system, no change in the concentration of nitrate was observed for both dark and irradiation experiments lasting up to 30 hours, irregardless of nitrate concentration, or type of titania. Sorption of nitrate on titania does not seem to occur in detectable quantities.

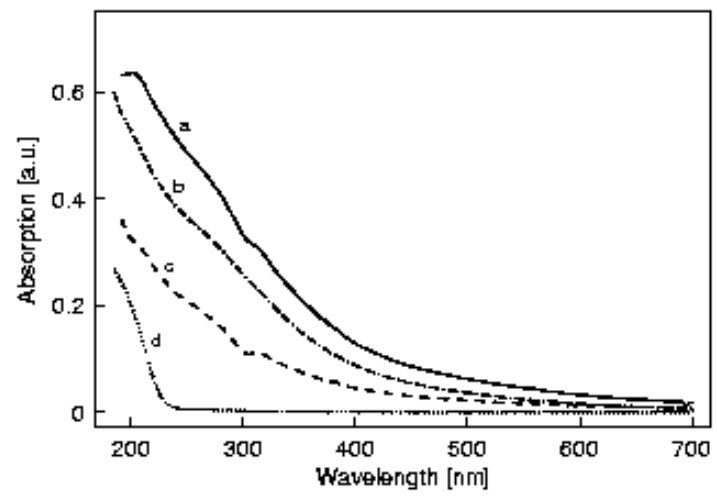

Figure 4: UV/Vis spectra of filtrates obtained from humic acids/titania mixtures after 30 hours of reaction: a) humic acids, not irradiated; b) humic acids, irradiated; c) humic acids and titania, not irradiated; d) humic acids and titania, irradiated. Humic acids $20 \mathrm{mg} / \mathrm{l}$; titania (P25) $0.1 \mathrm{~g} / \mathrm{l}$.

For the humic acid/titania system, UV/Vis spectra of the reaction mixture filtrates were taken after 30 hours of reaction, Figure 4. Spectra which were recorded after 30hour dark experiments showed no changes when Kronos1002 was used but a decrease in absorption in the range 200 to $500 \mathrm{~nm}$ was observed when either P25 (Figure 4c) or DT-51 were used, indicating sorption of the humic acids on these two titanias. The spectra taken after irradiation (Figure 4d) showed decreases in the absorptions attributed to humic acids, which were much more dramatic than the changes observed without irradiation or without titania, Figures 3 and 4.

\section{Three component system}

The nitrate concentration did not change in the dark experiments which were performed on the nitrate/humic acids/titania (any titania) systems. Changes in the nitrate concentration were not observed when mixtures of nitrate, humic acids, and DT-51 were irradiated. Nitrate conversions in the nitrate/humic acids/P25 system never exceeded $10 \%$, not even after 79 hours. Significant nitrate conversions, i.e. up to $32 \%$ after 76 hours, were reached in the nitrate/humic acids/Kronos-1002 system.

The nitrate concentration did not decrease monotonically with reaction time when the reaction was performed in the presence of Kronos-1002 (Figure 2). Considerable decrease in the nitrate concentration, i.e. $-31 \%$, was observed after only 3 hours. Nitrite was detected in concentrations as high as $5 \mathrm{mg} / \mathrm{l}$ during this period. The nitrate concentration after 30-50 hours was only about 4$11 \%$ lower than the initial concentration, with nitrite levels of $0-2 \mathrm{mg} / \mathrm{l}$. After 76 hours, nitrate concentrations had decreased by $30 \%$, indicating an overall trend towards higher conversion with longer time on stream. The nitrite concentration was $2 \mathrm{mg} / \mathrm{l}$ after 76 hours.

\section{Discussion}

\section{Photoinduced reduction of nitrate}

Early observations of the homogeneous photoreduction of nitrate to give nitrite were reported by Thiele [2]. Further investigations [3-6,30,31] yielded inconsistent results regarding a nitrate/nitrite equilibrium $[3,4]$ and regarding the product distribution which besides nitrite has been reported to include oxygen atoms [3,5], molecular oxygen [6,31], hydrogen peroxide [6], hydroxyl radicals [31], peroxynitrate [6], and pernitrite [31]. In Warneck's more recent investigations [7] two pathways of nitrate photodecomposition are suggested, one giving nitrite and oxygen atoms and one giving nitric oxide and oxygen radical anions. Kudo et al. [18] irradiated $1 \mathrm{M} \mathrm{NaNO}_{3}$ and $1 \mathrm{M} \mathrm{HNO}_{3}$, respectively, in experiments similar to ours and observed the formation of nitrite with a rate of $0.3 \mu \mathrm{mol} / \mathrm{h}$ in both cases. An increasing rate of formation of nitrite with increasing $\mathrm{pH}$ in the range of 5 to 8 was reported by Daniels [5], Wagner [6], Warneck [7], and Shuali [31]. Photoreduction of nitrate to nitrite has also been applied for the quantitative analysis of nitrate [32,33], with an increased reduction efficiency at increased $\mathrm{pH}$.

Consistent with the literature, nitrate in aqueous solution was observed to react under irradiation. The nitrate conversion was a function of time on stream, apparently approaching a limit at about 30\%, Figure 2. Nitrite concentrations reached the same order of magnitude as the amounts of nitrate converted, indicating that nitrite is a major product. The results show that it is extremely important to account for the homogeneous photoinduced reaction in the nitrate/water system when photocatalytic 
reactions are analyzed. It should also be noted that nitrite is more toxic than nitrate and thus a highly undesirable product. The successful nitrate decomposition process should either avoid the formation of nitrite, or convert the nitrite in fast secondary reactions to innocuous products.

\section{Humic Acids and Nitrate}

It has been reported in the literature [22-25] that humic acids are modified under irradiation. Oliver et al. [23] irradiated humic acids in aqueous medium and analyzed the products which were formed after scavengers had been added. Analysis of potential reaction pathways led the authors to conclude that species such as singlet oxygen, superoxide $\left(\mathrm{O}_{2}{ }^{-}\right)$, hydroxyl radicals, and $\mathrm{H}_{2} \mathrm{O}_{2}$ must have been present after the irradiation of the humic acids. Zepp et al. [22] used UV-absorption experiments to identify transients and inferred the formation of hydrated electrons from humic acids after laser flash photolysis. Vinodgobal et al. [24] also observed the formation of solvated electrons in a similar experiment conducted in the presence of $\mathrm{ZnO}$. Based on the results of Oliver [23], Vinodgobal [24], and Zepp [22], the formation of various reactive species can be assumed, some of which may be able to act as scavengers for oxygen atoms formed in the photoreduction of nitrate and thus may prevent a backreaction. Alternatively, some species, e.g. solvated electrons, may themselves reduce nitrate through a homogeneous, stoichiometric reaction. Nitrate is known to be an electron scavenger [22].

We observed changes in the UV spectra of humic acids after irradiation. Changes occurred in the regime of 200 to $400 \mathrm{~nm}$. An assignment is not possible because humic acids have a wide variety of functional groups, no welldefined bands are observed, and scattering effects contribute to the spectrum. Our data only allow confirmation of an interaction between photons and humic acids. Reactions of functional groups under irradiation continued for 30 hours as shown by decreasing absorption with irradiation time (Figure 3). The spectra demonstrate that even after 30 hours of irradiation UV-Vis active functional groups are present suggesting that reactive species are also available at long reaction times.

Reaction between nitrate and humic acids without irradiation was not detected. The photoinduced conversion of nitrate in the presence of humic acids was always faster than in the absence of humic acids (Figure 2) despite lower transmittance through the reaction medium than through the nitrate solutions, particularly in the range of high photon energy (200 to $400 \mathrm{~nm}$, Figures 1,3,4). Nitrite was the major product, and was formed in amounts equivalent to the amounts of nitrate converted. A maximum nitrate conversion of $45 \%$ was approached after 70 hours when typical reaction conditions were applied. The degradation of the UV/Vis-absorbing functional groups of the humic acids under irradiation was not accelerated in a detectable manner when nitrate was present.

The positive influence of the humic acids on the conversion of nitrate could have its origin in the increase of the $\mathrm{pH}$ upon addition of the humic acids. Other authors [3,6,31] observed an increase in the rate of formation of nitrite with increased $\mathrm{pH}$. It is not possible to distinguish from our data whether the effect of the humic acids is a consequence of the change in $\mathrm{pH}$, or of reactive species formed from humic acids under irradiation, i.e. their functioning as scavengers or reducing agents.

\section{Humic Acids and Titania}

UV-light is necessary to induce the transition of an electron from the valence band into the conduction band of large band gap semiconductors such as titania (band gap $3.29 \mathrm{eV}$ or $385 \mathrm{~nm}$ for anatase). Electrons from excited states of adsorbed molecules, typically dyes which are excited by light in the visible region, can be transferred into the conduction bands of semiconductors [8]. These transferred electrons on the semiconductor surface are then available for reduction of other adsorbates. If humic acids provide electrons to titania, then their interaction with the semiconductor (titania) surface would be a prerequisite. The adsorption of humic acids on titania was suspected already by Fox [34]. Vinodgobal investigated the charge transfer from fulvic acid (a humic substance) to zinc oxide [24] and from humic acids to titania [25]. Charge injection from Suwanee River Humic Acid to colloidal titania in an acetonitrile/water mixture was successful, so that reduction of oxazine dyes with titania was accomplished [25].

We observed the sorption of humic acids on two different titanias, i.e. DT-51 and P25. The surface area of Kronos1002 may be too small to adsorb a large enough fraction of humic acids to give a measurable effect in the UV-Vis spectrum of the aqueous phase after removal of the titania. The decrease of UV-absorptions from humic acids after irradiation in the presence of titania is much larger than the changes observed for irradiation only or for sorption on titania without irradiation. Thus, the interaction of humic acids with the titania surface is influenced by irradiation. On the basis of Vinodgobal's results, an electron transfer from the humic acids to titania is expected, providing electrons on the titania surface for reduction reactions. Regeneration of the humic acid would require another electron donor; but in our system further oxidation seems more likely, because titania, particularly anatase [23], is a photocatalyst for oxidation reactions [11-13,35], e.g. the oxidation of alcohols, carboxylic acids or chlorine containing pollutants. Consistent with oxidative degradation of humic acids are the decreasing absorptions in the UV/Vis spectra. Humic acids participate as a stoichiometric reagent rather than as a co-catalyst.

\section{Nitrate and Titania}

Photoreduction of nitrate in the presence of titania was investigated by Kudo et al. [18,19] who found small amounts of nitrite as a product, the rate of formation being of the same order of magnitude as without the catalyst. Reduction of nitrate to ammonia could only be achieved with platinum or nickel oxide promoted titania. Ammonia was the predominant product in acidic solution, and nitrite in alkaline solution; nitrite was identified as an intermediate 
of ammonia formation. Ranjit et al. [17,36-38] studied the reduction of nitrate employing a series of photocatalysts, among them ruthenium promoted titania. The product was ammonia, and nitrite could not be detected as an intermediate, although nitrite itself could also be reduced to ammonia. Neither nitrate nor nitrite could be reduced to ammonia without the ruthenium promoter. Reduction of nitrite in an aqueous suspension of titania without metal promoters was found to be possible in the presence of sulfide [39].

Nitrite can also be reoxidized to nitrate with oxygen present and with the anatase phase of titania as a photocatalyst as shown by Hori et al. [40]. Zafra et al. [41] reported that the yield of the photooxidation of nitrite and the amount of nitrite adsorbed on titania increased considerably with decreasing $\mathrm{pH}$. Zafra's results were consistent with Langmuir-Hinshelwood kinetics. Even nitrogen $\left(\mathrm{N}_{2}\right)$ can be fixed and oxidized on titania (rutile) surfaces $[42,43]$.

The addition of titania thus leads to several effects which are disadvantageous for the photoreduction of nitrate: The transmittance of the reaction medium is reduced. The $\mathrm{pH}$ is less than 6, which slows the homogeneous reduction. Reoxidation to nitrate proceeds easily if oxygen is present; in our open system we would expect oxygen to be dissolved in the reaction medium although some salting out is expected due to the potassium nitrate. The fact that we did not observe any changes in the nitrate concentration suggests that if reduction occurs at all, reoxidation is fast.

\section{Nitrate, Humic Acids, and Kronos-1002 Titania}

Interpretation of the results of the three component system is difficult because of the number of parameters and the number of possible interactions. Homogeneous reduction of nitrate with the formation of nitrite will always occur, as will the reoxidation of nitrite to form nitrate on titania if oxygen is present. The $\mathrm{pH}$ has been identified as an important factor, with the homogeneous reduction of nitrate to nitrite being favorable at high $\mathrm{pH}$, and the sorption and oxidation of nitrite on titania being favorable at low $\mathrm{pH}$. The reaction slurry has a medium $\mathrm{pH}$ depending on the type of titania and the titania/humic acid ratio. Humic acids will react under irradiation, they will adsorb and react on the titania surface, and they might be consumed by these reactions.

We observed considerable nitrate conversion at short reaction times (3-6 hours), with the formation of nitrite. However, after 30 hours of reaction the nitrate concentration was found to be only a few percent less than the initial concentration. After 70 hours of reaction time the nitrate concentration was again significantly reduced and of the same order of magnitude as in the nitrate and nitrate/humic acids system, but apparently there was less nitrite, indicating that there might be other reaction pathways. However, care must be taken in comparing conversions at the same reaction time in different reaction media due to variations in transmittance.
The combination of humic acids and titania accelerates the conversion of nitrate in the initial stage of the reaction. The seemingly low conversion of nitrate at 30 hours can be explained by that fact that the predominant product is nitrite so that the back reaction is possible. Since rapid conversion is observed only in the initial stage, stoichiometric rather than catalytic reactions may occur, i.e. the humic acids may be consumed. At long reaction times, the nitrate concentration in the 3-component system approaches values comparable to those in the nitrate and the nitrate/humic acid system (see Figure 2).

\section{Conclusions}

The homogeneous photoreduction of nitrate to form nitrite is a non negligible reaction in the photocatalytic decomposition of nitrate, particularly at $\mathrm{pH}$ values greater than 6 to 8 . The formation of nitrite is undesirable, because it is more toxic than nitrate.

Titania alone did not appear to catalyze the photoreduction of nitrate; however, nitrite is easily reoxidized to nitrate on the titania surface when oxygen is present, particularly at low $\mathrm{pH}$. Without the addition of humic acids, once nitrite is formed, the reoxidation pathway seems to prevail over a further reduction to nitrogen or ammonium.

Humic acids promoted the reduction of nitrate. Humic acids can undergo numerous reactions under irradiation, homogeneous reactions as well as reactions on the titania surface. These reactions may be stoichiometric as well as catalytic, and products would have to be analyzed before humic acids could enter a process for drinking water purification. The positive effect of humic acids on nitrate conversion may be in part due to the $\mathrm{pH}$ increase caused by the humic acids.

In systems containing nitrate, humic acids, and titania, the nitrate concentrations after long reaction times (70 hours) equaled concentrations observed when pure nitrate or nitrate and humic acids were irradiated; however, the amount of nitrite formed is less than the amount of nitrate converted and thus other reaction pathways were significant. The complexity of the system is clear from the initially high conversion of nitrate to nitrite and the subsequent recovery of the nitrate concentration followed by a further decrease of the nitrate concentration without an equivalent increase in the nitrite concentration.

\section{Acknowledgements}

We thank F. Willig and J. Mahrt (Hahn-Meitner-Institute, Berlin) for kindly providing the light source. R. Nießner (Technical University, Munich) and R. Schöllhorn (Technical University, Berlin) are gratefully acknowledged for their resourceful contributions. We thank M. Thiede for technical assistance and J. Schütze for the surface area measurements. 


\section{References}

[1] U. Rohmann, H. Sontheimer, Nitrat im Grundwasser, DVGW-Forschungsstelle am Engler- Bunte-Institut der Universität Karlsruhe, Karlsruhe, 1985, p. 280.

[2] H. Thiele, Ber. 40 (1907) 4914.

[3] W.T. Anderson, J. Am. Chem. Soc. 46 (1924) 797, and references therein.

[4] D.S. Villars, J. Am. Chem. Soc. 49 (1927) 326.

[5] M. Daniels, R.V. Meyers, E.V. Belardo, J. Phys. Chem. 72 (1968) 389.

[6] I. Wagner, H. Strehlow, G. Busse, Z. Phys. Chem. N. F. 123 (1980) 1.

[7] P. Warneck, C. Wurzinger, J. Phys. Chem. 92 (1988) 6278.

[8] A.L. Linsebigler, G. Lu, J.T. Yates Jr., Chem. Rev. 95 (1995) 735.

[9] O. Legrini, E. Oliveros, A.M. Braun, Chem. Rev. 93 (1993) 671.

[10] D. Bahnemann, Nachr. Chem. Tech. Lab. 42 (1994) 378.

[11] R.W. Matthews, Wat. Res. 24 (1990) 653.

[12] U. Stafford, K.A. Gray, P.V. Kamat, J. Catal. 167 (1997) 25.

[13] D. Bahnemann, D. Bockelmann, R. Golisch, Sol. Ener. Mat. 24 (1991) 564.

[14] C. Richard, New. J. Chem. 18 (1994) 443.

[15] C. Minero, E. Pelizzetti, P. Piccinini, M. Vincenti, Chemosphere 28 (1994) 1229.

[16] M.W. Rophael, M.A. Malati, J. Chem. Soc., Chem. Commun. (1987) 1418.

[17] K.T. Ranjit, T.K. Varadarajan, B. Viswanathan, J. Photochem. Photobiol. A 89 (1995) 67.

[18] A. Kudo, K. Domen, K. Maruya, T. Onishi, J. Catal. 135 (1992) 300.

[19] A. Kudo, K. Domen, K. Maruya, T. Onishi, Chem. Lett. (1987) 1019.

[20] W. Ziechmann, Huminstoffe, Verlag Chemie, Weinheim, 1980, p. 8.

[21] J. Falbe, M. Regitz (Eds.), Römpp Chemie Lexikon, Georg Thieme Verlag, Stuttgart, 1995. Vol. 3, p. 1866.

[22] R.G. Zepp, A. Braun, J. Hoigné, J.A. Leenheer, Environ. Sci. Technol. 21 (1987) 485.

[23] B. G. Oliver, J.H. Carey in E. Pelizzetti, N. Serpone (Eds.), Homogeneous and Heterogeneous Photocatalysis, Reidel Publishing Company, Dordrecht, 1986, p. 629.

[24] K. Vinodgobal, P.V. Kamat, Environ. Sci. Technol. 26 (1992) 1963.

[25] K. Vinodgopal, Res. Chem. Intermed. 20 (1994) 825.

[26] J. Lawrence in B.K. Afghan, A.S.Y. Chau (Eds.), Analysis of Trace Organics in the Aquatic Environment, CRC Press Inc., Boca Raton, 1989, p. 313.

[27] M. Ettlinger, Schriftenreihe Pigmente: Hochdisperse Metalloxide nach dem Aerosil ${ }^{\circledR}$ Verfahren, Nr. 56, Degussa, 1980, p. 13-14.

[28] C.A. Parker, Proc. Roy. Soc. (London) A 220 (1953) 104.

[29] G. Jander, E. Blasius, Lehrbuch der analytischen und präparativen anorganischen Chemie, S. Hirzel Verlag, Stuttgart, 1983, p. 241.

[30] N.S. Bayliss, R.B. Bucat, Aust. J. Chem. 28 (1975) 1865.

[31] U. Shuali, M. Ottolenghi, J. Rabani, Z. Yelin, J. Phys. Chem. 73 (1969) 3445.

[32] K. Takeda, K. Fujiwara, Anal. Chim. Act. 276 (1993) 25.

[33] S. Motomizu, M. Sanada, Anal. Chim. Act. 308 (1995) 406.

[34] Fox, see question and answer section in reference 20.

[35] J. Theurich, M. Lindner, D.W. Bahnemann, Langmuir 12 (1996) 6368.

[36] K.T. Ranjit, T.K. Varadarajan, B. Viswanathan, Ind. J. Chem. 35A (1996) 177.

[37] K.T. Ranjit, R. Krishnamoorthy, T.K. Varadarajan, B. Viswanathan, J. Photochem. Photobiol. A: Chem. 86 (1995) 185.

[38] K.T. Ranjit, R. Krishnamoorthy, B. Viswanathan, J. Photochem. Photobiol. A: Chem. 81 (1994) 55.

[39] M. Halmann, K. Zuckerman, J. Chem. Soc. Chem. Comm. (1986) 455.

[40] Y. Hori, A. Nakatsu, S. Suzuki, Chem. Lett. (1985) 1429.

[41] A. Zafra, J. Garcia, A. Milis, X. Domènech, J. Mol. Catal. 70 (1991) 343.

[42] R.I. Bickley, V. Vishwanathan, Nature 280 (1979) 306.

[43] J.A. Navio, C. Cerillos, C. Real, Surf. Interf. Anal. 24 (1996) 355. 